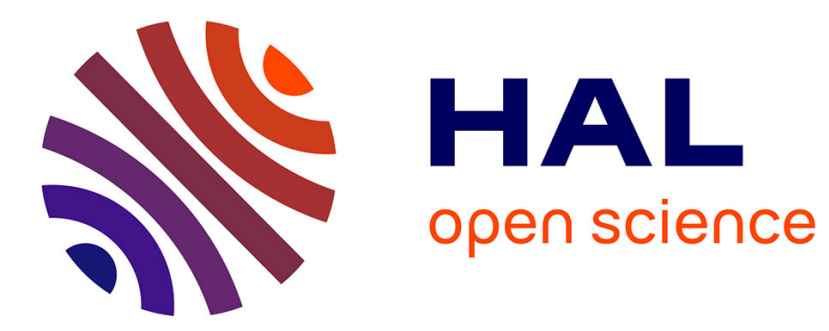

\title{
Delay Analysis of IEEE 802.15.6 CSMA/CA Mechanism in Duty-Cycling WBANs
}

Rongrong Zhang, Hassine Moungla, Mehaoua Ahmed

\section{To cite this version:}

Rongrong Zhang, Hassine Moungla, Mehaoua Ahmed. Delay Analysis of IEEE 802.15.6 CSMA/CA Mechanism in Duty-Cycling WBANs. IEEE Global Communications Conference 2015, Dec 2015, San

Diego, United States. hal-01270450

\section{HAL Id: hal-01270450 \\ https://hal.science/hal-01270450}

Submitted on 7 Feb 2016

HAL is a multi-disciplinary open access archive for the deposit and dissemination of scientific research documents, whether they are published or not. The documents may come from teaching and research institutions in France or abroad, or from public or private research centers.
L'archive ouverte pluridisciplinaire HAL, est destinée au dépôt et à la diffusion de documents scientifiques de niveau recherche, publiés ou non, émanant des établissements d'enseignement et de recherche français ou étrangers, des laboratoires publics ou privés. 


\title{
Delay Analysis of IEEE 802.15.6 CSMA/CA Mechanism in Duty-Cycling WBANs
}

\author{
Rongrong Zhang, Hassine Moungla, Ahmed Mehaoua \\ LIPADE, University of Paris Descartes, Sorbonne Paris Cité 45 rue des saints pères, 75006, Paris, France \\ Email: \{rongrong.zhang; hassine.moungla; ahmed.mehaoua\}@ parisdescartes.fr
}

\begin{abstract}
Duty-cycle has recently attracted significant research attention due to its paramount importance on energy conservation in Wireless Body Area Networks (WBANs). However, the additional delay resulted from applying duty-cycle is overlooked in most, if not all, of existing work, despite the fundamental importance of the delay in healthcare applications. In order to bridge this gap, we devote this paper to analyzing the delay of IEEE 802.15.6 CSMA/CA mechanism under duty-cycle. Technically, we first explicitly formulate the expressions of the random delay that a sensor node spends on transmitting packets under asynchronous duty-cycling protocol of IEEE 802.15.6 CSMA/CA. Moreover, we mathematically derive the probabilistic characteristics in terms of the expectation and variance of the delay. Furthermore, we conduct elaborate simulations to demonstrate the correctness of the theoretical analysis.
\end{abstract}

\section{INTRODUCTION}

Wireless Body Area Networks (WBANs) which offer numerous highly promising applications in the fields of healthcare, entertainment and military have attracted considerable attention [1]. To address the medium access (MAC) and physical layer (PHY) needs of low-power and short-range WBANs, IEEE Task Group TG6 was established in November 2007 to realize a standard, i.e., IEEE 802.15.6, whose final version was released in February 2012 [2]. Recently, there have been a steady stream of research efforts on the performance analysis of IEEE 802.15.6 CSMA/CA and the design of energy-efficient protocols for WBANs.

Analysis of IEEE 802.15.6 CSMA/CA [3-6]. The authors in [3] derived the maximum throughput and minimum delay bounds of IEEE 802.15.6 CSMA/CA for different data rates and frequency bands in the system with one sender and one receiver for an ideal channel with no transmission errors and no collision. Subsequently, the mean response time, reliability and throughput of IEEE 802.15.6 CSMA/CA under saturated and non-saturated traffic regime are developed by Markov model in [4] and [5], respectively. Moreover, the effect of user priorities on the performance of IEEE 802.15.6 in terms of the throughput and average packet delay for the medical traffic is evaluated in [6].

Duty-cycling protocols [7-8]. By implementing duty-cycle, the nodes can periodically switch between active and sleep states to save energy. The energy-efficient and reliable communication protocol in [7] is able to achieve energy conservation and collision avoid by low duty-cycle and Time Division Multi Access (TDMA) approaches. Furthermore, the nodes can dynamically adjust the wake-up and sleep modes according to their traffic to solve the idle listening and overhearing problems to save energy in [8].

However, most of the existing work either analyzes the performance with ideal channel with no bit error rate and no collision or does not take into account the additional delay resulted from duty-cycle of IEEE 802.15.6 CSMA/CA despite its great importance in healthcare applications.

Motivated by the above observations, we argue that a systematic study on the effect of duty-cycle on the delay of IEEE 802.15.6 CSMA/CA is called for in order to lay the theoretical foundations for the design and optimization of protocols for WBANs. To that end, we devote this paper to analyze the transmission delay of IEEE 802.15.6 CSMA/CA in duty-cycling WBANs.

The main technical contributions of this paper are articulated as follows. We first explicitly formulate the random delay that a sensor node spends on transmitting packets under asynchronous duty-cycling protocol of IEEE 802.15.6 CSMA/CA. Moreover, we derive the probabilistic characteristics in terms of the expectation and variance of the delay. Furthermore, we validate the theoretical results by simulations. To the best of our knowledge, our work is the first theoretical analysis on the delay of combining IEEE 802.15.6 CSMA/CA together with duty-cycle for WBANs.

The remainder of this paper is organized as follows. Section II introduces two fundamental analytical mechanisms and the problem formulation. In section III, we detailly analyze the random delay of one successful communication between one sensor and the sink. In Section IV, the theoretical expressions of the delay are validated by simulation and numerical results. Finally, we conclude our paper in section V.

\section{Analytical Mechanism and Problem Statement}

\section{A. Analytical Mechanism}

In this subsection, we outline CSMA/CA mechanism of IEEE 802.15.6 under asynchronous duty-cycle for investigating the delay performance later. In the mechanism, we consider a generic WBAN topology where sensor nodes (or simply called nodes) with the same user priority communicate with the sink by single hop. The sink and nodes are duty-cycled independently, and their duty-cycle periods are decided by the network traffic and the packet arrival rate, respectively.

The main communication procedure can be summarized as follows. Once the sink wakes up, it first broadcasts a burst of preambles by employing the received-initiated Low Power 
Listening (LPL) and IEEE 802.15.6 CSMA/CA mechanism, as will be described later. When the nodes with pending packets wake up and receive one preamble successfully, they will send back an ACK following CSMA/CA mechanism. After succeeding to receive the ACK, the sink remains awake to receive the subsequent packets. But if there are no active sensor nodes during the maximum duration of the sink broadcasting preambles, the sink will turn to sleep. Figure 1 is an illustration of the communication procedure.

Low Power Listening (LPL), also referred to as preamble sampling, is a key technique used by a large number of asynchronous duty-cycling MAC protocols to save energy. LPL mechanism can be divided into two categories: senderinitialed LPL [1] and receiver-initialed LPL [9].

Compared with the sender-initiated LPL, the receiverinitiated LPL is more appropriate for low-power and energy limited WBANs [9], as the sink can be more battery unconstrained or be more possible to recharge or replace the exhausted batteries than the nodes. In receiver-initiated LPL, the sink triggers a burst of short preambles periodically and waits for the feedback from the nodes. When the sensor nodes wake up and receive the preambles, they reply with ACKs and then transmit packets.

IEEE 802.15.6 CSMA/CA is different from IEEE 802.15.4 CSMA/CA for its special back-off mechanism [2]. In IEEE 802.15.6 CSMA/CA, a node should maintain three variables for each transmission attempt: $N B, C W$ and $B C$. $N B$ is the number of back-off times; $C W$ is the value of contention window; and $B C$ is the back-off counter.

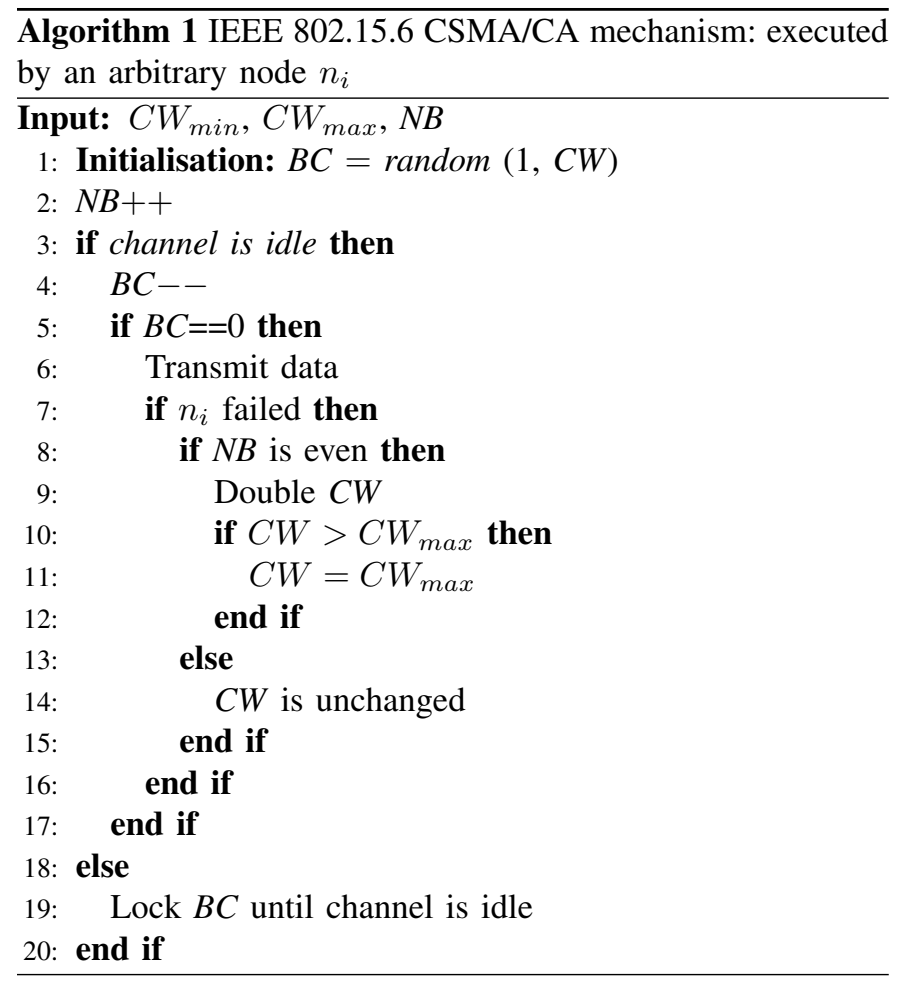

A formal description of IEEE 802.15.6 CSMA/CA protocol is shown in Algorithm 1 for an arbitrary node. First, after sensing the channel is idle for a Short Inter-Frame Spacing (SIFS) time interval, a node initializes its $B C$ to a random integer uniformly distributed over the interval $[1, C W]$, where $C W \in\left(C W_{\min }, C W_{\max }\right)$. The values of $C W_{\min }$ and $C W_{\max }$ vary depending on the priority of a user. Meanwhile, the number of $N B$ increases by one for each attempt. Subsequently, if the channel is idle, the node decreases $B C$ by one. Otherwise, the node locks its $B C$ until the channel is idle again. Furthermore, when the channel is idle and $B C$ reaches zero, the node can send a packet. If the transmission fails, $C W$ is doubled but should be not greater than $C W_{\max }$ for even $N B$, while $C W$ remains unchanged for odd $N B$. The node then returns to initialize $\mathrm{BC}$ and repeats the back-off procedure.

\section{B. Problem Formulation}

Given a duty-cycling WBAN of one sink and $N$ sensor nodes, our objective is to accurately analyze the performance of IEEE 802.15.6 CSMA/CA mechanism under asynchronous duty-cycle. By accurately we mean that the theoretical results match well the simulation results. Moreover, the performance analyzed in this paper is the delay that a node experiences to transmit a data packet, definitely, the probabilistic characteristics of the delay: the probability mass function, the expectation and variance.

\section{Delay AnAlysis}

In the analysis, we explicitly formulate the delay $T_{d}$ which is defined as the expected time between the packet arrival at a node and its reception at the sink. We derive the delay $T_{d}$ by calculating the following components:

- $T_{1}$ : random delay spent by the sink before the transmission of a preamble.

- $T_{w}$ : random delay spent by the node from the instant of data packet arrival until the successful reply to the sink with an ACK.

- $T_{\text {data }}$ : time spent by the node on transmitting packets.

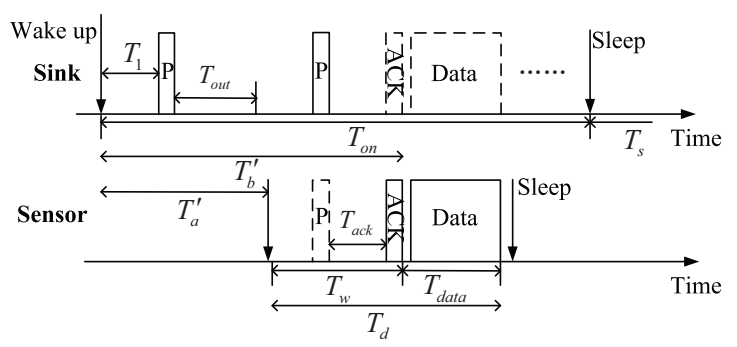

Fig. 1. Illustration of communication between a sensor and the sink

As shown in Fig.1, the delay to successfully send a data packet is thus $T_{d}=T_{w}+T_{\text {data }}$. In the following, we formally formulate these delay components in sequence.

\section{A. Modeling of $T_{1}$}

Assume that the mechanism to transmit a preamble for the sink is the same with an ACK transmission for sensor nodes as specified in IEEE 802.15.6 CSMA/CA protocol. Let $\alpha$ be the probability of busy channel. Let $N_{b}$ be the maximum number of back-off of a preamble, i.e., the number of times 
that the sink attempts to access the channel before giving up the transmission of the preamble.

Let $t_{p, k, j}$ be the random back-off time before the $j$ th attempt for the case where the channel is busy for the $k-1$ times and free at the $k$ th time. It follows that $t_{p, k, j}$ has a uniform distribution over the interval $[1, C W] \cdot S_{b}$, where $S_{b}$ is a unit back-off period. Denote by $\mathscr{A}_{k}$ the event that the channel is busy for the $k-1$ times and free at the $k$ th time, and by $\mathscr{A}$ the event that a preamble is backed off at most $N_{b}$ times. Thus, random delay $T_{1}$ spent by the sink before transmitting a preamble within $N_{b}$ attempts can be expressed as

$$
T_{1}=\sum_{k=1}^{N_{b}}\left(\sum_{j=1}^{k} t_{p, k, j}\right) \mathbf{1}_{\mathscr{A}_{k} \mid \mathscr{A}}=\sum_{k=1}^{N_{b}} \Sigma_{k} \mathbf{1}_{\mathscr{A}_{k} \mid \mathscr{A}},
$$

where $\mathbf{1}_{(\cdot)}$ is the indicator function $\left(\mathbf{1}_{(\cdot)}=1\right.$ if the argument is true, and $\mathbf{1}_{(\cdot)}=0$ otherwise) and $\Sigma_{k}=\sum_{j=1}^{k} t_{p, k, j}$ is a random variable describing the time spent on the $k$ th back-off. Consequently, we can obtain the probabilistic characteristics as stated in the following lemma 1.

Lemma 1. The mean and variance of $T_{1}$ are

$$
\begin{aligned}
& \mu_{T_{1}}=\mathbb{E} T_{1}=\sum_{k=1}^{N_{b}} \mu_{\Sigma_{k}} \frac{\alpha^{k-1}}{\sum_{j=1}^{N_{b}} \alpha^{j-1}}, \\
& \sigma_{T_{1}}^{2}=\mathbb{E}\left[T_{1}-\mathbb{E}\left[T_{1}\right]\right]^{2}=\sum_{k=1}^{N_{b}} \sigma_{\Sigma_{k}}^{2} \frac{\alpha^{k-1}}{\sum_{j=1}^{N_{b}} \alpha^{j-1}},
\end{aligned}
$$

where $\mathbb{E}[\cdot]$ defines the mean of a random variable and

$$
\begin{aligned}
& \mu_{\Sigma_{k}}=\mathbb{E}\left[\Sigma_{k}\right]=\sum_{j=1}^{k} \mu_{t_{p, k}, j}, \\
& \sigma_{\Sigma_{k}}^{2}=\mathbb{E}\left[\Sigma_{k}-\mathbb{E}\left[\Sigma_{k}\right]\right]^{2}=\sum_{j=1}^{k} \sigma_{t_{p, k}, j}^{2},
\end{aligned}
$$

where $\mu_{t_{p, k, j}}$ and $\sigma_{t_{p, k, j}}^{2}$ denote the mean and variance of the random variable $t_{p, k, j}$ which is uniformly distributed in the interval $[1, C W] \cdot S_{b}$.

Proof: Since $\mathscr{A}_{k}$ is the event that the channel is busy for the $k-1$ times and free at the $k$ th time, the probability of $\mathscr{A}_{k}$ is $\operatorname{Pr}\left(\mathscr{A}_{k}\right)=\alpha^{k-1}(1-\alpha)$, where $\alpha$ is assumed to be independent at each attempt. The probability of $\mathscr{A}$ that a preamble is backed off at most $N_{b}$ times is then derived as

$$
\operatorname{Pr}(\mathscr{A})=\operatorname{Pr}\left(\sum_{j=1}^{N_{b}} \mathscr{A}_{j}\right)=\sum_{j=1}^{N_{b}} \operatorname{Pr}\left(\mathscr{A}_{j}\right)
$$

where the equality comes from that the events $\mathscr{A}_{j}$, $j=1, \ldots, N_{b}$ are mutually exclusive. It thus also holds that

$$
\begin{aligned}
\operatorname{Pr}\left(\mathscr{A}_{k} \mid \mathscr{A}\right) & =\frac{\operatorname{Pr}\left(\mathscr{A}_{k} \sum_{j=1}^{N_{b}} \mathscr{A}_{j}\right)}{\operatorname{Pr}(\mathscr{A})}=\frac{\operatorname{Pr}\left(\mathscr{A}_{k}\right)}{\sum_{j=1}^{N_{b}} \operatorname{Pr}\left(\mathscr{A}_{j}\right)} \\
& =\frac{\alpha^{k-1}}{\sum_{j=1}^{N_{b}} \alpha^{j-1}}
\end{aligned}
$$

Notice that $\Sigma_{k}$ is given by the sum of independent uniformly distributed random variables. Therefore, the mean of $\Sigma_{k}$ is given by

$$
\mu_{\Sigma_{k}}=\mathbb{E}\left[\Sigma_{k}\right]=\sum_{j=1}^{k} \mu_{t_{p, k, j}}
$$

where $\mu_{t_{p, k, j}}=(C W-1) S_{b} / 2$. And the variance of $\Sigma_{k}$ is thus given by the sum of the variance of $t_{p, k, j}$ hence

$$
\sigma_{\Sigma_{k}}^{2}=\mathbb{E}\left[\Sigma_{k}-\mathbb{E}\left[\Sigma_{k}\right]\right]^{2}=\sum_{j=1}^{k} \sigma_{t_{p, k}, j}^{2}
$$

where $\sigma_{t_{p, k, j}}^{2}=(C W-1)^{2} S_{b}^{2} / 12$. By using $\mu_{\Sigma_{k}}$ and $\sigma_{\Sigma_{k}}^{2}$ and applying the properties of the expectation operator, the lemma 1 follows.

Remark. Since $T_{1}$ is the weighted sum of uniform random variables with different mean and variance, no closed-form expression is available for the probability mass function. However, a Gaussian distribution can approximate the probability mass function of $T_{1}$.

In addition, since the ACK message is transmitted with the same mechanism of the preamble, the mean and variance of the random delay $T_{a c k}$ spent by sensor nodes before sending back an ACK to the sink can be approximated as

$$
\begin{aligned}
\mu_{T_{a c k}} & =\sum_{k=1}^{N_{b}} \mu_{\Sigma_{k}} \frac{\alpha^{k-1}}{\sum_{k=1}^{N_{b}} \alpha^{k-1}}, \\
\sigma_{T_{a c k}}^{2} & =\sum_{k=1}^{N_{b}} \sigma_{\Sigma_{k}}^{2} \frac{\alpha^{k-1}}{\sum_{k=1}^{N_{b}} \alpha^{k-1}} .
\end{aligned}
$$

\section{B. Modeling of $T_{w}$}

In this subsection, we analyze $T_{w}$ that the random delay between the wake-up moment of a sensor node and the time when an ACK is sent successfully to the sink during $T_{\text {out }}$, where $T_{\text {out }}$ is defined as the maximum time that the sink waits for an ACK message after sending a preamble. Due to the implementation of asynchronous duty-cycling protocol, we proceed to derive $T_{w}$ in the following two cases:

$$
T_{w}= \begin{cases}T_{a}+T_{b}, & \text { Case } 1: \text { Sink is sleeping } \\ T_{b}^{\prime}-T_{a}^{\prime}, & \text { Case } 2: \text { Sink is awake. }\end{cases}
$$

Case 1: The sink is sleeping when the sensor node is awake and intends to transmit packets, as shown in Fig.2.

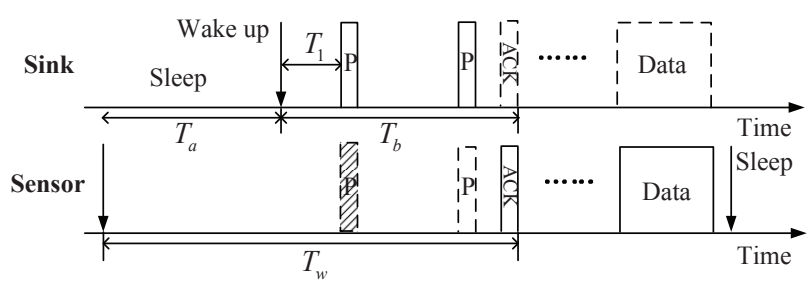

Fig. 2. Modeling of $T_{w}$ in case 1

First, denote by $T_{a}$ the random time between the wake-up of a node and that of the sink. $T_{a}=0 \mathbf{1}_{\overline{\mathscr{B}}}+T_{2} \mathbf{1}_{\mathscr{B}}$, where the event $\mathscr{B}$ occurs when the sink is sleeping and nodes have packets to transmit, and $T_{2}$ is the random time to wait for the wake-up 
of the sink given that the event $\mathscr{B}$ is true. Consequently, the probabilities of $\mathscr{B}$ and $\overline{\mathscr{B}}$ are

$$
\operatorname{Pr}(\mathscr{B})=\frac{T_{s}}{T_{\text {on }}+T_{s}}, \quad \operatorname{Pr}(\overline{\mathscr{B}})=1-\operatorname{Pr}(\mathscr{B}),
$$

where $T_{s}$ and $T_{o n}$ are the duration of the sleep and activation of the sink during one duty-cycle period, respectively. Furthermore, since $T_{2}$ is a uniform distribution in the range $\left[0, T_{s}\right]$, the probability mass function of $T_{a}$ is

$$
\operatorname{Pr}\left(T_{a}\right)= \begin{cases}\frac{1}{T_{o n}+T_{s}} & 0<T_{a} \leq T_{s} \\ \frac{T_{o n}}{T_{o n}+T_{s}} & T_{a}=0\end{cases}
$$

We then define $T_{b}$ as the time interval from the wake-up of the sink until a node sends an ACK during $T_{\text {out }}$ after receiving one preamble given that the event $\mathscr{B}$ is true, as shown in Fig.2.

In order to avoid that the sink spends much energy on transmitting preambles as there is no incoming packets for long time, the maximum duration to transmit preambles for the sink is restrained as $T_{p}$. Denote by $N_{p}$ the maximum number of preambles that the sink can sent during $T_{p}$. If there is no feedback from nodes after $T_{p}$, the sink will turn to sleep. And define $\mathscr{C}_{k}$ as the event that the sink has to send $k$ preambles before being received and the node responds with an ACK which is then received successfully by the sink. Now we can formulate $T_{b}$ as

$$
T_{b}=\sum_{k=1}^{N_{p}}\left(\sum_{j=1}^{k} T_{1, k, j}+(k-1) T_{\text {out }}+T_{a c k}\right) \mathbf{1}_{\mathscr{C}_{k} \mid \mathscr{C}}
$$

where $T_{1, k, j}$ is the random delay of the transmission of the $j$ th preamble when the $k$ th preamble with the distribution given in Eq.(1) is received successfully. And $T_{a c k}$ is the delay spent by a node on sending an ACK successfully to the sink. $\mathscr{C}$ is the event that the sink receives an ACK within $N_{p}$ preambles. We next present the probabilistic characteristics of $T_{b}$ in lemma 2 .

Lemma 2. The mean and variance of $T_{b}$ are

$$
\begin{aligned}
& \mu_{T_{b}}=\sum_{k=1}^{N_{p}}\left(k \mu_{T_{1}}+(k-1) T_{\text {out }}+\mu_{T_{\text {ack }}}\right) \frac{\operatorname{Pr}\left(\mathscr{C}_{k}\right)}{\sum_{j=1}^{N_{p}} \operatorname{Pr}\left(\mathscr{C}_{j}\right)}, \\
& \sigma_{T_{b}}^{2}=\sum_{k=1}^{N_{p}} \sigma_{T_{b, k}}^{2} \frac{\operatorname{Pr}\left(\mathscr{C}_{k}\right)}{\sum_{j=1}^{N_{p}} \operatorname{Pr}\left(\mathscr{C}_{j}\right)},
\end{aligned}
$$

where $\sigma_{T_{b, k}}^{2}$ is the variance of $\sum_{j=1}^{k} T_{1, k, j}+(k-1) T_{\text {out }}+$ $T_{a c k}$ and

$\operatorname{Pr}\left(\mathscr{C}_{k}\right)=\left[\operatorname{Pr}\left(k T_{1}+(k-1) T_{\text {out }}\right) \leq T_{p}\right)\left(1-\operatorname{Pr}\left(T_{\text {ack }} \leq T_{\text {out }}\right)\right)$

$$
\begin{aligned}
& \left.\times \operatorname{Pr}\left(T_{\text {ack }} \leq T_{\text {out }}\right) \operatorname{Pr}(\overline{\mathscr{P}})\right] \\
& +\left[\operatorname{Pr}\left(k T_{1}+(k-1) T_{\text {out }}\right) \leq T_{p}\right) \\
& \left.\times \operatorname{Pr}\left(T_{\text {ack }} \leq T_{\text {out }}\right)^{2} \operatorname{Pr}(\mathscr{P}) \operatorname{Pr}(\mathscr{P})\right] .
\end{aligned}
$$

Proof: The proof is based on expressing the mean and variance of $T_{b}$ in terms of $\operatorname{Pr}\left(\mathscr{C}_{k}\right)$. According to the expression of $T_{b}$ in Eq.(3), its mean and variance can be presented as

$$
\begin{aligned}
\mu_{T_{b}} & =\sum_{k=1}^{N_{p}}\left(k \mu_{T_{1}}+(k-1) T_{\text {out }}+T_{\text {ack }}\right) \operatorname{Pr}\left(\mathscr{C}_{k} \mid \mathscr{C}\right), \\
\sigma_{T_{b}}^{2} & =\sum_{k=1}^{N_{p}} \sigma_{T_{b, k}}^{2} \operatorname{Pr}\left(\mathscr{C}_{k} \mid \mathscr{C}\right) .
\end{aligned}
$$

Using the fact that the events $\mathscr{C}_{j}, j=1, \ldots, N_{p}$ are mutually exclusive, we have

$$
\operatorname{Pr}(\mathscr{C})=\operatorname{Pr}\left(\sum_{k=1}^{N_{p}} \mathscr{C}_{k}\right)=\sum_{k=1}^{N_{p}} \operatorname{Pr}\left(\mathscr{C}_{k}\right),
$$

and then

$$
\operatorname{Pr}\left(\mathscr{C}_{k} \mid \mathscr{C}\right)=\frac{\operatorname{Pr}\left(\mathscr{C}_{k} \sum_{j=1}^{N_{p}} \mathscr{C}_{j}\right)}{\operatorname{Pr}(\mathscr{C})}=\frac{\operatorname{Pr}\left(\mathscr{C}_{k}\right)}{\sum_{j=1}^{N_{p}} \operatorname{Pr}\left(\mathscr{C}_{j}\right)},
$$

which completes the proof.

Next, the key step is to find the expression for $\operatorname{Pr}\left(\mathscr{C}_{k}\right)$. To that end, we firstly define $\tau$ as the probability of nodes attempting to transmit in a randomly chosen time slot. And denote by $\mathscr{P}$ the event of losing a preamble or an ACK due to a collision. Thus $\operatorname{Pr}(\overline{\mathscr{P}})=1-(1-\tau)^{N-1}$ is the probability that at least one of the remaining nodes attempts to transmit in the same time slot, where $N$ is the number of nodes. As the size of a preamble and an ACK is much smaller than that of data packets, we assume that these probabilities are independent at each attempt.

Furthermore, we denote $\mathscr{D}_{k}$ as the event that the sink transmits $k$ preambles before the expiration of $T_{p}$. Denote by $\mathscr{F}_{k}$ the event that an ACK is transmitted successfully in the case that $\mathscr{D}_{k}$ is true. Let $\Omega$ be the certain event. Now, we are ready to determine $\operatorname{Pr}\left(\mathscr{C}_{k}\right)$ in lemma 3 .

Lemma 3. Following the definition of $\mathscr{C}_{k}$, it holds that

$$
\mathscr{C}_{k}=\left(\mathscr{D}_{k-1} \overline{\mathscr{F}}_{k-1}+\mathscr{D}_{k-1} \mathscr{F}_{k-1} \mathscr{P}\right) \mathscr{D}_{k} \mathscr{F}_{k} \overline{\mathscr{P}}
$$

where

$$
\begin{aligned}
& \left.\mathscr{D}_{0}=\Omega, \quad \mathscr{D}_{k}=\left(k T_{1}+(k-1) \boldsymbol{1}_{(k-1) \geq 0} T_{\text {out }}\right) \leq T_{p}\right), \\
& \overline{\mathscr{F}}_{0}=\left(T_{\text {ack }}>T_{\text {out }}\right), \quad \mathscr{\mathscr { F }}_{k}=\left(T_{\text {ack }} \leq T_{\text {out }} \mid \mathscr{D}_{k}\right), \\
& \overline{\mathscr{F}}_{k-1}=\left(T_{\text {ack }}>T_{\text {out }} \mid \mathscr{D}_{k-1} \boldsymbol{1}_{(k-1) \geq 0}\right) .
\end{aligned}
$$

Proof:

From the definition of $\mathscr{C}_{k}, \mathscr{C}_{k}$ consists of two events: 1) the event $\mathscr{C}_{1, k}=\mathscr{D}_{k-1} \overline{\mathscr{F}}_{k-1} \mathscr{D}_{k} \mathscr{F}_{k} \overline{\mathscr{P}}$ occurs when the $k-1$ th preamble was sent but the nodes were not able to send back an ACK before the timeout; and 2) the event $\mathscr{C}_{2, k}=$ $\mathscr{D}_{k-1} \mathscr{F}_{k-1} \mathscr{P} \mathscr{D}_{k} \mathscr{F}_{k} \overline{\mathscr{P}}$ occurs when the $k-1$ th preamble was sent and an ACK was sent back before the timeout but it was collided. Note that $\mathscr{C}_{1, k} \mathscr{C}_{2, k}=\phi$, we thus have

$$
\operatorname{Pr}\left(\mathscr{C}_{k}\right)=\operatorname{Pr}\left(\mathscr{C}_{1, k}\right)+\operatorname{Pr}\left(\mathscr{C}_{2, k}\right)
$$

Subsequently, we derive the probabilities of $\mathscr{C}_{1, k}$ and $\mathscr{C}_{2, k}$. By considering that the events of $\mathscr{F}_{k}$ and $\mathscr{P}$ are independent of the others, the probability of $\mathscr{C}_{1, k}$ is given as

$$
\operatorname{Pr}\left(\mathscr{C}_{1, k}\right)=\operatorname{Pr}\left(\mathscr{D}_{k-1} \mathscr{D}_{k}\right) \operatorname{Pr}\left(\overline{\mathscr{F}}_{k-1} \mathscr{F}_{k}\right) \operatorname{Pr}(\overline{\mathscr{P}})
$$

for $\mathscr{D}_{k-1} \mathscr{D}_{k}=\mathscr{D}_{k}$. Note that $\overline{\mathscr{F}}_{k-1}$ and $\mathscr{F}_{k}$ are independent, and that $\operatorname{Pr}\left(\overline{\mathscr{F}}_{k-1}\right)=1-\operatorname{Pr}\left(\mathscr{F}_{k-1}\right)=1-\operatorname{Pr}\left(T_{\text {ack }} \leq T_{\text {out }}\right)$, we thus have

$$
\begin{aligned}
& \operatorname{Pr}\left(\mathscr{C}_{1, k}\right)=\operatorname{Pr}\left(\mathscr{D}_{k}\right) \operatorname{Pr}\left(\overline{\mathscr{F}}_{k-1}\right) \operatorname{Pr}\left(\mathscr{F}_{k}\right) \operatorname{Pr}(\overline{\mathscr{P}}) \\
& \left.=\operatorname{Pr}\left(k T_{1}+(k-1) T_{\text {out }}\right) \leq T_{p}\right)\left(1-\operatorname{Pr}\left(T_{\text {ack }} \leq T_{\text {out }}\right)\right) \\
& \times \operatorname{Pr}\left(T_{\text {ack }} \leq T_{\text {out }}\right) \operatorname{Pr}(\mathscr{\mathscr { P }}) .
\end{aligned}
$$

Furthermore, since $\mathscr{D}_{k-1} \mathscr{D}_{k}=\mathscr{D}_{k}$ and $\mathscr{F}_{k-1}$ is independent of $\mathscr{F}_{k}$, it holds that

$$
\mathscr{D}_{k-1} \mathscr{F}_{k-1} \mathscr{P}_{k} \mathscr{F}_{k} \overline{\mathscr{P}}=\mathscr{F}_{k-1} \mathscr{P} \mathscr{D}_{k} \mathscr{F}_{k} \overline{\mathscr{P}} \text {. }
$$


As a consequence, it holds that

$$
\begin{aligned}
\operatorname{Pr}\left(\mathscr{C}_{2, k}\right)= & \operatorname{Pr}\left(\mathscr{F}_{k-1}\right) \operatorname{Pr}(\mathscr{P}) \operatorname{Pr}\left(\mathscr{D}_{k}\right) \operatorname{Pr}\left(\mathscr{F}_{k}\right) \operatorname{Pr}(\overline{\mathscr{P}}) \\
= & \left.\operatorname{Pr}\left(k T_{1}+(k-1) T_{\text {out }}\right) \leq T_{p}\right) \\
& \times \operatorname{Pr}\left(T_{\text {ack }} \leq T_{\text {out }}\right)^{2} \operatorname{Pr}(\mathscr{P}) \operatorname{Pr}(\mathscr{P}) .
\end{aligned}
$$

Substituting Eq.(6)-(7) into Eq.(5) yields $\operatorname{Pr}\left(\mathscr{C}_{k}\right)$ in case 1.

As a result, the random delay in case 1 is $T_{w}=T_{a}+T_{b}$.

Case 2: The sink is awake when sensor nodes have packets to transmit, as shown in Fig.1.

Inspired by the analysis in case 1 , let $T_{a}^{\prime}$ be the time interval between the wake-up instants of the sink and nodes. From the definition of $\mathscr{B}$, it holds that $T_{a}^{\prime}=0 \mathbf{1}_{\mathscr{B}}+T_{3} \mathbf{1}_{\mathscr{B}}$, where $T_{3}$ is the random time spent by the sink on waiting for the wake-up of a sensor node given that the event $\mathscr{B}$ is false. Since $T_{3}$ has a uniform distribution in the range $\left[0, T_{p}\right]$, the probability mass function of $T_{a}^{\prime}$ is

$$
\operatorname{Pr}\left(T_{a}^{\prime}\right)= \begin{cases}\frac{T_{o n}}{\overline{T_{p}\left(T_{o n}+T_{s}\right)}} & 0<T_{a}^{\prime} \leq T_{p}, \\ \overline{T_{s}} & T_{a}^{\prime}=0 .\end{cases}
$$

Correspondingly, let $T_{b}^{\prime}$ denote the random delay spent by the sink from its wake-up until it sends back an ACK successfully before the timeout of the sink given that the event $\overline{\mathscr{B}}$ is true. From the definition of $\mathscr{C}_{k}$ and referring to the derivation of $T_{b}, T_{b}^{\prime}$ can be formulated as

$$
T_{b}^{\prime}=\sum_{k=1}^{N_{p}}\left(\sum_{j=1}^{k} T_{1, k, j}+(k-1) T_{\text {out }}+T_{a c k}\right) \mathbf{1}_{\mathscr{C}_{k} \mid \mathscr{C}} .
$$

Note that the occurrence of $\mathscr{C}_{k}$ in case 2 is different from that in case 1 since the sink does not know when nodes wake up. Although the expression of $T_{b}^{\prime}$ is the same with $T_{b}$, the expressions of $\operatorname{Pr}\left(\mathscr{C}_{k}\right)$ in cases 1 and 2 differ from each other, as shown in the following lemma 4.

Lemma 4. In case $2, \operatorname{Pr}\left(\mathscr{C}_{k}\right)$ is derived as

$$
\begin{aligned}
\operatorname{Pr}\left(\mathscr{C}_{k}\right) & =\left(\operatorname{Pr}\left(\mathscr{N}_{k} \mathscr{D}_{k}\right)-\operatorname{Pr}\left(\overline{\mathscr{E}}_{k}\right)\right) \operatorname{Pr}\left(\mathscr{F}_{k}\right) \operatorname{Pr}(\overline{\mathscr{P}}) \\
& +\left(\operatorname{Pr}\left(\mathscr{D}_{k}\right)-\operatorname{Pr}\left(\mathscr{N}_{k} \mathscr{D}_{k}\right)\right) \operatorname{Pr}\left(\overline{\mathscr{F}}_{k-1}\right) \operatorname{Pr}\left(\mathscr{F}_{k}\right) \operatorname{Pr}(\overline{\mathscr{P}}) \\
& +\left(\operatorname{Pr}\left(\mathscr{D}_{k}\right)-\operatorname{Pr}\left(\mathscr{N}_{k} \mathscr{D}_{k}\right)\right) \operatorname{Pr}\left(\mathscr{F}_{k-1}\right) \operatorname{Pr}\left(\mathscr{F}_{k}\right) \operatorname{Pr}(\mathscr{P}) \\
& \times \operatorname{Pr}(\overline{\mathscr{P}}),
\end{aligned}
$$

where

$$
\begin{aligned}
\operatorname{Pr}\left(\overline{\mathscr{E}}_{k}\right) & =P_{1}\left(\frac{T_{a}^{\prime}-(k-1) T_{\text {out }}}{k}\right), \\
\operatorname{Pr}\left(\mathscr{D}_{k}\right) & =P_{1}\left(\frac{T_{p}-(k-1) T_{\text {out }}}{k}\right), \\
\operatorname{Pr}\left(\mathscr{N}_{k}\right) & =P_{1}\left(\frac{T_{a}^{\prime}-(k-2) T_{\text {out }}}{k-1}\right), \\
\operatorname{Pr}\left(\mathscr{N}_{k} \mathscr{D}_{k}\right) & =\operatorname{Pr}\left(\mathscr{D}_{k}\right)\left(1-\operatorname{Pr}\left(T_{1} \leq T_{p}-T_{a}^{\prime}-T_{\text {out }}\right)\right) \\
& +\operatorname{Pr}\left(\mathscr{N}_{k}\right) \operatorname{Pr}\left(T_{1} \leq T_{p}-T_{a}^{\prime}-T_{\text {out }}\right) .
\end{aligned}
$$

Proof: Let $\mathscr{N}_{k}$ define the event occurring when the $k-1$ th preamble is sent but the node is sleeping. And the event $\mathscr{E}_{k}$ occurs when the $k$ th preamble is sent and the node is awake. Recall the definitions in case 1 , we then have

$\mathscr{C}_{k}=\left(\mathscr{N}_{k}+\mathscr{E}_{k-1} \mathscr{D}_{k-1} \overline{\mathscr{F}}_{k-1}+\mathscr{E}_{k-1} \mathscr{D}_{k-1} \mathscr{F}_{k-1} \mathscr{P}\right) \mathscr{E}_{k} \mathscr{D}_{k} \mathscr{F}_{k} \overline{\mathscr{P}}$ where

$$
\begin{aligned}
\mathscr{N}_{k} & =\left((k-1) \mathbf{1}_{(k-1) \geq 0} T_{1}+(k-2) \mathbf{1}_{(k-2) \geq 0} T_{\text {out }} \leq T_{a}^{\prime}\right), \\
\mathscr{E}_{k} & =\left(k T_{1}+(k-1) \mathbf{1}_{(k-1) \geq 0} T_{\text {out }}>T_{a}^{\prime}\right) .
\end{aligned}
$$

In case 2, a preamble fails in three situations, which follows that $\mathscr{C}_{k}=\mathscr{C}_{1, k}+\mathscr{C}_{2, k}+\mathscr{C}_{3, k}$, where $\mathscr{C}_{1, k}=\mathscr{N}_{k} \mathscr{E}_{k} \mathscr{D}_{k} \mathscr{F}_{k} \mathscr{P}$ is the event that the node was sleeping when the sink sent the $k-1$ th preamble; $\mathscr{C}_{2, k}=\mathscr{E}_{k-1} \mathscr{D}_{k-1} \overline{\mathscr{F}}_{k-1} \mathscr{E}_{k} \mathscr{D}_{k} \mathscr{F}_{k} \bar{P}$ is the event occurring when the $k-1$ th ACK was not sent back before the timeout; and $\mathscr{C}_{3, k}=\mathscr{E}_{k-1} \mathscr{D}_{k-1} \mathscr{F}_{k-1} \mathscr{P}_{\mathscr{E}} \mathscr{D}_{k} \mathscr{F}_{k} \overline{\mathscr{P}}$ is the event occurring when the $k-1$ th ACK sent back before the timeout was collided. For $\mathscr{C}_{1, k} \mathscr{C}_{2, k}=\phi, \mathscr{C}_{1, k} \mathscr{C}_{3, k}=\phi$ and $\mathscr{C}_{2, k} \mathscr{C}_{3, k}=\phi$, we have

$$
\operatorname{Pr}\left(\mathscr{C}_{k}\right)=\operatorname{Pr}\left(\mathscr{C}_{1, k}\right)+\operatorname{Pr}\left(\mathscr{C}_{2, k}\right)+\operatorname{Pr}\left(\mathscr{C}_{3, k}\right) .
$$

As a consequence, the key step next is to derive the probabilities of $\mathscr{C}_{1, k}, \mathscr{C}_{2, k}$ and $\mathscr{C}_{3, k}$.

For the mutual independence of the event $\mathscr{F}_{k}$ and $\mathscr{P}$, the probability of $\mathscr{C}_{1, k}$ is given by

$$
\operatorname{Pr}\left(\mathscr{C}_{1, k}\right)=\operatorname{Pr}\left(\mathscr{N}_{k} \mathscr{E}_{k} \mathscr{D}_{k}\right) \operatorname{Pr}\left(\mathscr{F}_{k}\right) \operatorname{Pr}(\overline{\mathscr{P}}) .
$$

Following the total probability theorem, we have

$$
\operatorname{Pr}\left(\mathscr{N}_{k} \mathscr{D}_{k}\right)=\operatorname{Pr}\left(\mathscr{N}_{k} \mathscr{E}_{k} \mathscr{D}_{k}\right)+\operatorname{Pr}\left(\mathscr{N}_{k} \overline{\mathscr{E}}_{k} \mathscr{D}_{k}\right),
$$

and it holds that $\mathscr{N}_{k} \overline{\mathscr{E}}_{k} \mathscr{D}_{k}=\overline{\mathscr{E}}_{k} \mathscr{D}_{k}=\overline{\mathscr{E}}_{k}$ such that

$$
\operatorname{Pr}\left(\mathscr{N}_{k} \mathscr{E}_{k} \mathscr{D}_{k}\right)=\operatorname{Pr}\left(\mathscr{N}_{k} \mathscr{D}_{k}\right)-\operatorname{Pr}\left(\overline{\mathscr{E}}_{k}\right)
$$

Rewriting $\mathscr{N}_{k}=k T_{1}+(k-1) T_{\text {out }} \leq T_{a}^{\prime}+T_{1}+T_{\text {out }}$, we have

$$
\mathscr{N}_{k} \mathscr{D}_{k}= \begin{cases}\mathscr{D}_{k} & \text { if } T_{p} \leq T_{a}^{\prime}+T_{1}+T_{\text {out }}, \\ \mathscr{N}_{k} & \text { otherwise }\end{cases}
$$

whereby we get

$$
\begin{aligned}
\operatorname{Pr}\left(\mathscr{N}_{k} \mathscr{D}_{k}\right)= & \operatorname{Pr}\left(\mathscr{D}_{k}\right)\left(1-\operatorname{Pr}\left(T_{1} \leq T_{p}-T_{a}^{\prime}-T_{\text {out }}\right)\right) \\
& +\operatorname{Pr}\left(\mathscr{N}_{k}\right) \operatorname{Pr}\left(T_{1} \leq T_{p}-T_{a}^{\prime}-T_{\text {out }}\right),
\end{aligned}
$$

and combining this equation with Eq.(10) yields

$$
\operatorname{Pr}\left(\mathscr{C}_{1, k}\right)=\left(\operatorname{Pr}\left(\mathscr{N}_{k} \mathscr{D}_{k}\right)-\operatorname{Pr}\left(\overline{\mathscr{E}}_{k}\right)\right) \operatorname{Pr}\left(\mathscr{F}_{k}\right) \operatorname{Pr}\left(\overline{\mathscr{P}}_{)}\right.
$$

To compute $\operatorname{Pr}\left(\mathscr{C}_{2, k}\right)$, observe that

$$
\mathscr{E}_{k-1} \mathscr{D}_{k-1} \overline{\mathscr{F}}_{k-1} \mathscr{E}_{k} \mathscr{D}_{k} \mathscr{F}_{k} \overline{\mathscr{P}}=\mathscr{E}_{k-1} \mathscr{D}_{k} \overline{\mathscr{F}}_{k-1} \mathscr{F}_{k} \overline{\mathscr{P}},
$$

because $\mathscr{E}_{k-1} \mathscr{E}_{k}=\mathscr{E}_{k-1}=\overline{\mathscr{N}}_{k}$ and $\mathscr{D}_{k-1} \mathscr{D}_{k}=\mathscr{D}_{k}$. And note that $\overline{\mathscr{F}}_{k-1}$ and $\mathscr{F}_{k}$ are independent, so

$$
\begin{aligned}
& \operatorname{Pr}\left(\mathscr{C}_{2, k}\right)=\operatorname{Pr}\left(\overline{\mathscr{N}}_{k} \mathscr{D}_{k}\right) \operatorname{Pr}\left(\overline{\mathscr{F}}_{k-1}\right) \operatorname{Pr}\left(\mathscr{F}_{k}\right) \operatorname{Pr}(\overline{\mathscr{P}}) \\
& =\left(\operatorname{Pr}\left(\mathscr{D}_{k}\right)-\operatorname{Pr}\left(\mathscr{N}_{k} \mathscr{D}_{k}\right)\right) \operatorname{Pr}\left(\overline{\mathscr{F}}_{k-1}\right) \operatorname{Pr}\left(\mathscr{F}_{k}\right) \operatorname{Pr}(\overline{\mathscr{P}}) .
\end{aligned}
$$

Similarly, we have

$$
\mathscr{E}_{k-1} \mathscr{D}_{k-1} \mathscr{F}_{k-1} \mathscr{P}_{\mathscr{E}_{k}} \mathscr{D}_{k} \mathscr{F}_{k} \overline{\mathscr{P}}=\mathscr{E}_{k-1} \mathscr{D}_{k} \mathscr{F}_{k-1} \mathscr{P} \mathscr{F}_{k} \overline{\mathscr{P}} .
$$

Correspondingly, we can derive that

$$
\begin{aligned}
\operatorname{Pr}\left(\mathscr{C}_{3, k}\right)= & \operatorname{Pr}\left(\overline{\mathscr{N}}_{k} \mathscr{D}_{k}\right) \operatorname{Pr}\left(\mathscr{F}_{k-1}\right) \operatorname{Pr}(\mathscr{P}) \operatorname{Pr}\left(\mathscr{F}_{k}\right) \operatorname{Pr}(\overline{\mathscr{P}}) \\
= & \left(\operatorname{Pr}\left(\mathscr{D}_{k}\right)-\operatorname{Pr}\left(\mathscr{N}_{k} \mathscr{D}_{k}\right)\right) \operatorname{Pr}\left(\mathscr{F}_{k-1}\right) \operatorname{Pr}\left(\mathscr{F}_{k}\right) \\
& \times \operatorname{Pr}(\mathscr{P}) \operatorname{Pr}(\mathscr{P}) .
\end{aligned}
$$

Incorporating Eq.(11),(12),(13) leads to $\operatorname{Pr}\left(\mathscr{C}_{k}\right)$ in case 2.

Consequently, the delay in case 2 is $T_{w}=T_{b}^{\prime}-T_{a}^{\prime}$. In summary, since $T_{w}$ is given by the weighted sum of variables approximated as Gaussian distributed in Sect. III-A, it follows that $T_{w}$ can also be approximated by a Gaussian random variable. 


\section{Modeling of $T_{\text {data }}$}

The delay to transmit one date packet is approximated as

$$
T_{\text {data }}=L_{p} / R_{s}+T_{S I F S}+T_{h r}
$$

where $L_{p}$ is the payload of one packet in bits and $R_{s}$ is data rate in bits/s as in IEEE 802.15.6 [2]. $T_{S I F S}$ is the short interframe space interval among the separated data packet transmissions, and $T_{h r}$ is the time employed by the hardware platform to process the packets and propagate them.

Therefore, the delay for one successful communication between sink and a node is given by $T_{d}=T_{w}+T_{\text {data }}$, which is approximated by a Gaussian distribution as well, with mean $\mu_{T_{d}}=\mu_{T_{w}}+\mu_{T_{\text {data }}}$, and variance $\sigma_{T_{d}}^{2}=\sigma_{T_{w}}^{2}+\sigma_{T_{\text {data }}}^{2}$.

\section{Simulation Results}

In this section, we conduct extensive simulations to evaluate the accuracy of the theoretical results and illustrate the impact of duty-cycle on the delay. In the simulation, we consider a typical single-hop star WBAN of one sink and 10 nodes with the same user priority. Moreover, the parameters are set by following the specifications in the IEEE 802.15.6 standard [2], as listed in Table I.

TABLE I

SimUlation SETTINGS

\begin{tabular}{|c|c|c|c|}
\hline Parameter & Values & Parameter & Values \\
\hline$R_{s}$ & $242.9 \mathrm{kbps}$ & $L_{p}$ & 127 bytes \\
$S_{b}$ & $125 \mu \mathrm{s}$ & $C W_{\min }$ & 2 \\
$T_{h r}$ & $1 \mu \mathrm{s}$ & $C W_{\max }$ & 8 \\
$T_{S I F S}$ & $50 \mu \mathrm{s}$ & $N_{b}$ & 4 \\
$T_{p}$ & $0.1 T_{o n}$ & $N_{p}$ & 3 \\
\hline
\end{tabular}

Fig. 3 and Fig. 4 illustrate the analytic and emulational expectation and variance of the transmission delay $T_{d}$ as a function of different sleep time $T_{s}$ and active time $T_{o n}$, respectively. As shown in figures, the theoretical results match well with the simulation results, which prove the accuracy of the derived mathematical expression of the delay. Moreover, a good linear relationship between delay and the sleep time can be inferred from Fig. 3, especially in the cases that $T_{o n} \leq T_{s}$, since the packet transmission time and the active time are very short compared to the sleep time. Furthermore, the average delay decreases as the active time duration increases, but it is at the cost of more energy consumption.

\section{CONClusion}

In this paper, we have analyzed the transmission delay of IEEE 802.15.6 CSMA/CA in duty-cycling WBANs from the probability perspective. We first elaborated the receiverinitiated Low Power Listening (LPL) and IEEE 802.15.6 CSMA/CA mechanism. Then, we explicitly formulated the random delay spent by a sensor node on transmitting data packets. Subsequently, we derived the mean and variance of the delay as a function of the sleep time and active time. Finally, we conducted simulations and the results demonstrate the accuracy of theoretical analysis. In future work, we plan to design and optimize adaptive duty-cycling protocols for WBANs taking into account the tradeoff between the delay and energy consumption.

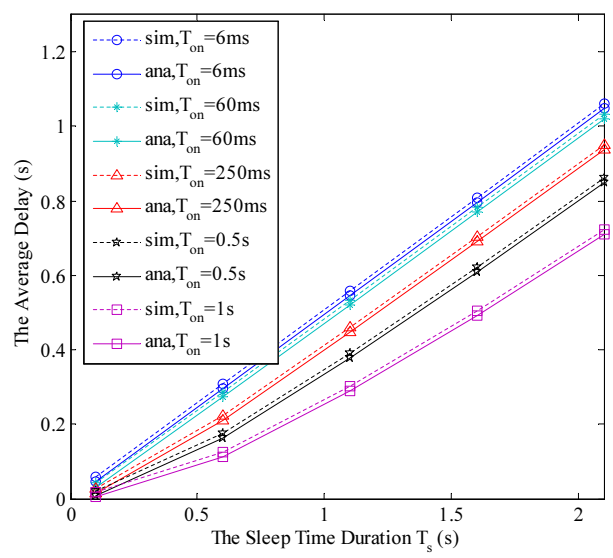

Fig. 3. The average delay to transmit a packet as obtained by analysis and simulations as a function of the sleep time $T_{s}$ for different active time $T_{o n}$

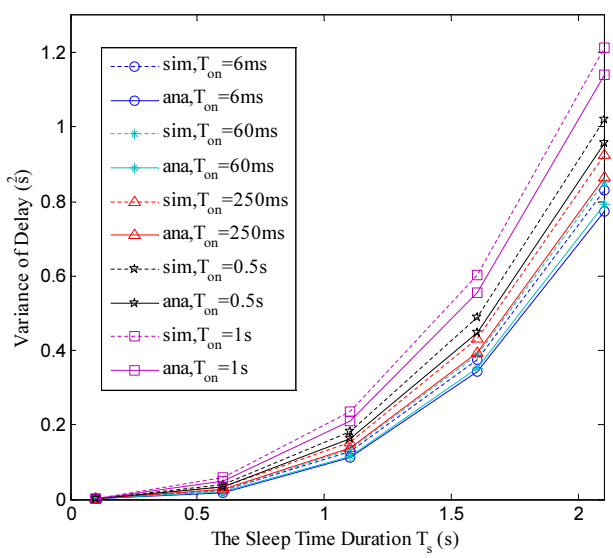

Fig. 4. The variance of the delay as obtained by analysis and simulations as a function of the sleep time $T_{s}$ for different active time $T_{o n}$

\section{REFERENCES}

[1] R. Cavallari, F. Martelli, and et al., "A survey on wireless body area networks: technologies and design challenges," IEEE Communications Surveys \& Tutorials, vol. 16, no. 3, pp. 1635-1657, 2014.

[2] A. Astrin and et al., "IEEE standard for local and metropolitan area networks - part 15.6: Wireless body area networks," pp. 1-271, 2012.

[3] S. Ulah, M. Chen, and K. S. Kwak, "Throughput and delay analysis of IEEE 802.15.6-based CSMA/CA protocol," Journal of Medical Systems, vol. 36, no. 6, pp. 3875-3891, 2012.

[4] S. Rashwand and J. Misic, "Performance evaluation of IEEE 802.15. 6 under non-saturation condition," in IEEE GLOBECOM, pp. 1-6, 2011.

[5] S. Sarkar, S. Misra, and et al., "Analysis of reliability and throughput under saturation condition of IEEE 802.15. 6 CSMA/CA for wireless body area networks," in IEEE GLOBECOM, pp. 2405-2410, 2014.

[6] L. Yang, C. Li, and et al., "Performance evaluation of IEEE 802.15. 6 MAC with user priorities for medical applications," in Ubiquitous Computing Application and Wireless Sensor, pp. 23-30, Springer, 2015.

[7] S. J. Marinkovic, E. M. Popovici, and et al., "Energy-efficient low duty cycle mac protocol for wireless body area networks," IEEE Trans. Info. Technol. in Biomed., vol. 13, no. 6, pp. 915-925, 2009.

[8] S. Ullah, "RFID-enabled MAC protocol for WBAN," in IEEE ICC, pp. 6030-6034, 2013.

[9] P. Dutta, S. Dawson-Haggerty, and et al, "Design and evaluation of a versatile and efficient receiver-initiated link layer for low-power wireless," in ACM SenSys, pp. 1-14, 2010. 